UNIOSUN Journal of Engineering and Environmental Sciences. Vol. 2, No. 2. Sept. 2020

DOI: 10.36108/ujees/0202.20.0231

\title{
Deployment of Hybrid Evolutionary Algorithm-Artificial Neural Network for Optimum Real Time Frequency Allocation in a Heterogeneous Frequency Requisition Network
}

\author{
Lasisi, H.O., Oyedeji, M.O., Oladepo, O. and Aderinkola, B.F.
}

\begin{abstract}
In heterogeneous frequency demand cellular networks, the frequency demand varies across the cells involved, in contrast to the homogeneous scenario, where the demand is the same. This paper reports the deployment of hybridized evolutionary algorithm and artificial neural network for optimum frequency allocation in heterogeneous frequency requisition mobile networks to ensure interference control. Frequency sharing and reuse among cells, due to scarcity, are fundamental in a communication network for optimum frequency utilization. Primarily, efficient frequency sharing allows cells of adequate reuse distance the utilization of the same channels, with minimized inter-cell interference. The degree of freedom from interference and efficient frequency allocation mechanism dictate the grade of service, GoS, of a communication network. A real-time frequency allocation is defined by some degree of randomness. Thus, frequency allotment problem is mostly expressed as a constrained optimization formulation. The optimal allocations are achieved at points of minimum cost metric. In this paper, the frequency allotment issue is expressed as a two-objective optimization challenge, using Key Performance Indicators (KPIs) data acquired via Drive Test as input parameters. NSGA-II, an evolutionary algorithm was first deployed on the formulated problem, then in combination with SOM, an artificial neural network technique. The hybrid algorithm was implemented in MATLAB for a heterogeneous frequency demand scenario. The results obtained from the hybrid technique show performance improvements of between $6 \%$ and $28 \%$ in terms of fitness indices for interference cost function and, between 3\% and $65 \%$ for demand infringement cost function. The algorithm could be embedded in the operating system of Base Station Controllers for enhanced real-time optimal allocation of network resources.
\end{abstract}

Keywords: Evolutionary Algorithm, Artificial Neural Network, Interference, Heterogeneous Demand, Grade of Service

\section{Introduction}

This work is an extension of the research work presented in [1], and therefore, focuses on the deployment of hybridized evolutionary algorithm and artificial neural network for optimum frequency allocation in a heterogeneous frequency requisition mobile network to ensuring interference control. Frequency sharing and reuse are important

Lasisi, H.O., Oyedeji, M.O., Oladepo, O. (Department of Electrical and Electronic Engineering, Osun State University, Osogbo, Nigeria)

Aderinkola, B.F. (Department of Electrical and Electronic Engineering, Federal Polytechnic, Ede, Nigeria) Corresponding author: hammed.lasisi@uniosun.edu.ng Telephone Number: +234-803-409-4040 planning mechanisms in communication engineering for effective resources utilization and coordination among the cells within a network. However, finest frequency reuse can be attained when the available frequency is effectively shared at minimized inter-cellular interference within the network. Moreover, Grade of Service, GoS, as a degree of the overall performance of a communication system, is a function of constructive frequency scheduling and allotment, which are distinguished by some degrees of arbitrariness. Consequently, Frequency allocation is generally formulated as constrained multiple objectives optimization issue involving complex algorithms, where the best possible 
solutions are achieved at points of least penalties.

Many researchers have worked on the application of evolutionary algorithms to wireless networking resource allocation problems. The related previous works are as presented in [1]. While many research works had efficiently deployed a particular optimization algorithm into solving resource allocation problems, some demonstrated, practically the general application of those algorithms. For example, [2], [3], [4], [5], [6], and [7] discussed the application of Genetic Algorithms to the allocation of resources in network systems, [8] dealt with the deployment of ant colony optimization technique. Applications of Non-dominated sorting genetic algorithms were discussed in [9] and [10]. However, multi-criteria optimization and multi-objective decision making are highlighted in [11] and [12] respectively.

Fundamentally, depending on the vision of a particular author, frequency allotment can be modelled as a multiple objective's optimization issue. This paper, as [1], modelled the allocation problem as a twoobjective optimization problem having two constraints. The objective functions are for the minimum interference and the total penalty incurred in a given frequency allotment. The evolutionary algorithm deployed in this work is non-dominated sorting genetic algorithm-II (NSGA-II). Advantages of NSGA-II include; lower computational complexity, faster than the conventional NSGA, non-requirement of specifying sharing parameter as crowded comparison approach is used, and production of better solutions via the principle of elitism [13]. NSGA-II achieves uniformly spread-out Pareto-optimal fronts and better solutions by ensuring comparison of a solution with all other solutions in the population. However, the major disadvantage of NSGA-II is that it has an increased storage requirement when compared with ordinary NSGA. In hybridization with NSGA-II, the brand of Artificial Neural Network (ANN) used in this work is the Self-organizing map (SOM). Selforganizing map is unsupervised, and so advantageous for its; simplicity and ease of understanding, high effectiveness in data classification, ability to reduce data dimensions with minimal or no loss of information, and its capability to learn from the data. Adversely, data classification provided by the SOM is a function of the user-defined map size, thus, various map sizes give different data classifications.

\section{Materials and Methods}

As in [1], the methodology employed involves engaging in drive tests to acquire data on mobile network key performance Indicators (KPIs) parameters. Drive tests can be done for network benchmarking, service quality monitoring, or optimization and troubleshooting depending on the desired results or problems to be solved. The model for predicting path loss developed by [14], in conjunction with measured parameters were used in evaluating the cell parameters required for the optimization problem formulation.

From [14], the model for path loss prediction for the research area was adopted to obtain the necessary input parameters required for optimization problem formulation. The procedure for the optimization problem formulation has three steps; the evaluation of cells' parameters, the objective functions formulation, and the institution of constraints. On the formulated problem, NSGA-II was first deployed, and later in combination with SOM for heterogeneous frequency demand scenarios. The algorithms were implemented 
in MATLAB, and the obtained results were compared for stand-alone NSGA-II and the hybrid algorithm.

To implement the hybrid algorithm on the formulated frequency allocation problem, a random population solution size of 100 was sorted into different levels of nondominations after creation. Offspring populations of the same size were then created via recombination and mutation operators. A SOM of map size $2 \times 2$ was then deployed to map the relationship in the newly generated individuals. This action resulted in dividing the new population into four subpopulations of similar phenotypes. The sub-populations were then evolved concurrently for several generations. The resulting sub-populations were combined and evolved for one more generation. Summarily, the methodology involved in this research work is herein broken down as;

\section{A. Cell Parameters Evaluation.}

The cell parameters required for estimation are the cell radius, the cell coverage area, the number of cells required for effective network coverage, and the appropriate cellular structure. A hexagonal cellular structure was assumed owing to the fact that it has best centre-to- centre distance, and gives no dead zone within the network coverage. Given that; the total area to be covered by network is represented by " $A$ ", " $R_{O}$ " is the cell radius estimated form deployment of the model for path loss prediction developed in [14], then, " $A_{C}$ ", the cell effective coverage area and " $N$ ", the number of required cells; as estimated in [1] are as given in Equations (1) and (2) respectively.

$A_{C}-\frac{6 R_{q}^{2}}{4} \cos 30^{\circ}$
$N=\frac{A}{A_{C}}$

\section{B. Problem Formulation.}

Here, as in [1], the frequency allotment problem was formulated on two objective functions: the minimum interference objective function and demand infringement penalty objective function. $\Psi$ represents a $V X F$ binary allocation matrix. $\rho(\Psi)$ denotes minimum interference function, while demand breach penalty function is represented by $d(\Psi)$. Therefore, the optimization requires finding an optimal frequency allocation that minimizes the two objective functions, when a set of cells represented by $V$ are to effectively share a group of frequencies represented by $F$. The combiner and the forbidden constraints are the two constraints guiding the formulation.

\section{Formulation of Interference Cost Function}

The interference cost function is formulated as expressed in Equation (3) [13];

$\min _{p} \rho(\psi)=\sum_{i \in v} \sum_{f \in F} \alpha_{i f},\left[\gamma_{i}(i, f)+\gamma_{a}(i, f)\right]$

Parameter, $\alpha_{i f}$ is the binary normalization factor. It exclusively assumes a binary value 1 or 0 . Its value is binary 1 if a cell is allotted a particular frequency; otherwise, it assumes the binary value 0 . The parameter $\gamma_{0}(i, f)$ represents co-channel interference and $\gamma_{a}(i, f)$ denotes adjacent channel interference. The binary normalization factor $\alpha_{i f}$ is defined as given in Equation (4);

$\sigma_{i f} \in\{0,1\}, \quad i \in V, \forall f \in F$

The co-channel interference $\gamma_{s}(i, f)$ and the adjacent channel interference $\gamma_{a}(i, f)$ in 
Equation (3) are $V \times F$ binary allocation matrices. Parameters $\gamma_{\sigma}(i, f)$ and $\gamma_{a}(i, f)$ are defined as in Equations (5) and (6) respectively;

$$
\begin{aligned}
& \gamma_{c}(i, f)=\sum_{j=0} w_{c}(i, j), \alpha_{j f} \forall i \in V, \forall f \in F
\end{aligned}
$$

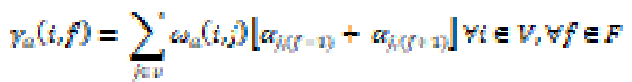

In Equation (5), the parameter $\omega_{o}\left(i_{j} j\right)$ is a matrix of $V$ number of rows and columns representing mutual interference values between cells sharing the same frequency. Moreover, in Equation (6), the parameter $\omega_{a}(i, j)$ is a matrix of $V$ number of rows and columns representing mutual interference values between cells using adjacent frequencies within the spectrum. Thus, the two parameters $\left(\omega_{g}\right.$ and $\left.\omega_{a}\right)$ can be expressed as in Equations (7) and (8).

$\omega_{c}=\left[\begin{array}{ccccc}\omega_{11}^{c} & \omega_{12}^{c} & \omega_{12}^{c} & \cdots & \omega_{1}^{c} \\ \omega_{11}^{c} & \omega_{2}^{c} & \omega_{n}^{c} & \cdots & \omega_{g}^{c} \\ \omega_{11}^{c} & \omega_{V}^{c} & \omega_{n}^{c} & \cdots & \omega_{V}^{c} \\ \vdots & \vdots & & \ddots & \vdots \\ \omega_{W 1}^{c} & \omega_{W 2}^{c} & \omega_{V 2}^{c} & \cdots & \omega_{W}^{c}\end{array}\right]$

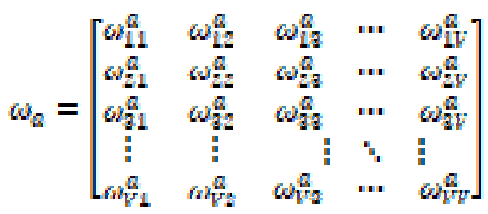

Considering the cell radius and re-use distance, the two parameters $\left(\omega_{g}\right.$ and $\left.\omega_{a}\right)$ are as given in Equations (9) and (10) respectively.

$$
\begin{aligned}
& \omega_{\sigma}(i, j)=32 \cdot R_{a}{ }^{4}[d(i, j)]^{-4} \\
& \omega_{n}(i, j)=4 . R_{n}{ }^{4}\lceil d(i, j)]^{-4}
\end{aligned}
$$

\section{Formulation of Demand Infringement Cost Function}

The demand infringement cost function is the sum of the penalties incurred if the frequency demands of the cells are not met. This is as given in Equation (11). $\min d(\Psi)=\sum_{i=\mathbb{V}} \beta_{i}$

Where " $\beta$ " represents the penalty value for a particular cell in the network and is given by equation (12). When the disparity between the frequency demand of a cell and the frequency allotted denoted by $\mathrm{n}$ is zero, " $\beta$ " has a value of zero; otherwise, it depends on a penalty parameter " $\mu$ ".

$$
\beta_{1}- \begin{cases}\mu^{-n,} & n<0 \\ 0, & n-0 \\ \mu^{n}, & n>0\end{cases}
$$

\section{E. Institution of the Constraints}

The combiner and the forbidden constraints are the two constraints guiding the formulation as well as the optimization involved. Observation of the essential guard band in frequency spectrum, and careful allocation of adjacent frequencies in the spectrum gave birth to the constraints. The constraints are as presented in Equations (13) and (14). $\lambda$ is the minimum separation for frequency reuse in the frequency spectrum.

$$
\begin{aligned}
& \left\|f \cdot \alpha_{i f}-g \cdot \alpha_{i g}\right\| \geq \lambda(i) \forall i \in V, \forall(f, g) \in F^{2} \\
& \left(\sum_{j \in \psi(b)} \alpha_{j f}+\sum_{j \in w(b)} a_{i f f-1)}+a_{j(f+1)}\right)=u \forall i \in v, \quad \forall f \in F
\end{aligned}
$$

The combiner constraint (as presented in Equation (13)) dictates that frequencies allotted to a cell must be adequately separated in the frequency spectrum to avoid intercellular interference. The forbidden constraint is as given in Equation (14). The constraint guides that it is forbidden for cells that are not adequately separated to be allotted the same or adjacent frequencies.

\section{Results and Discussion}

The performance of the hybrid evolutionary algorithm-artificial neural network for optimum real time frequency allocation in a 
heterogeneous frequency requisition network is evaluated. A total number of 14 cells were estimated for effective cellular network coverage in the studied area. The cells and the respective heterogeneous frequency demand are as presented in Table 1. However, Figure 1 and Figures 3-6 show the evolution of the stand-alone and hybrid algorithms respectively.On the formulated problem, NSGA-II was first deployed, and later in combination with SOM for heterogeneous frequency demand scenarios. The algorithms were implemented in MATLAB.
A random population solution size of 100 was sorted into different levels of nondominations after creation. Offspring populations of the same size were then created via recombination and mutation operators. Figure 1 presents Evolution of NSGA-II algorithm when deployed alone over 100 generations.

Table 1: The Cellular Network Heterogeneous Frequency Demand

\begin{tabular}{lllllllllllllllll}
\hline Cell Serial Number & 1 & 2 & 3 & 4 & 5 & 6 & 7 & 8 & 9 & 10 & 11 & 12 & 13 & 14 \\
\hline Frequency Demand of the Cell & 4 & 2 & 5 & 6 & 3 & 2 & 2 & 3 & 5 & 4 & 2 & 2 & 3 & 3 \\
\hline
\end{tabular}
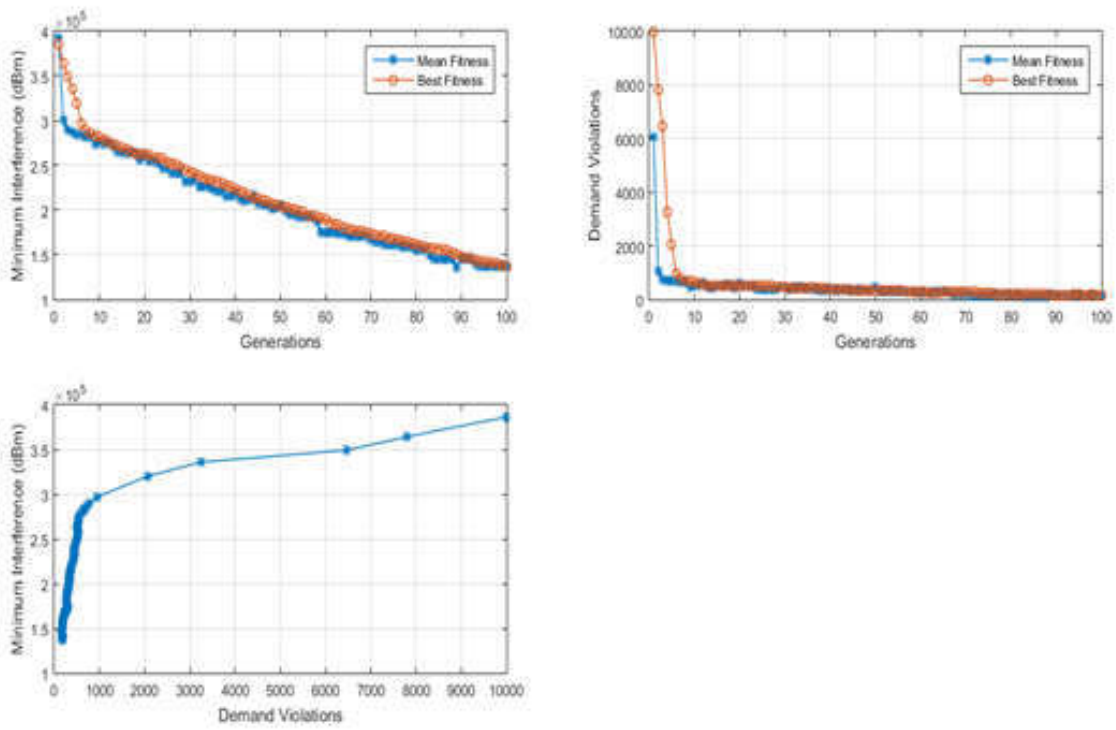

Figure 1: Evolution of the population when NSGA-II was deployed alone; (a) Evolution of the minimum interference; (b) Evolution of the demand infringement; (c) Combination of the minimum interference \& the demand infringement. 


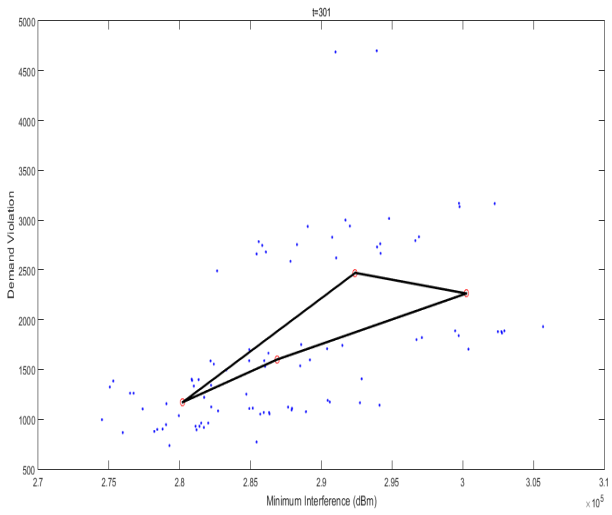

Figure 2: Division of the populations into four sub-populations by SOM

The outputs of the NSGA-II are nondominated solutions. The SOM was then deployed to map the relationship in the newly generated individuals. This action resulted in dividing the new population into four subpopulations of similar phenotypes. The sub-populations were then evolved concurrently for several generations. The resulting sub-populations were combined and evolved for one more generation. Figure 2 presents a self-organizing map dividing the populations into four sub-populations. The divisions (into sub-populations) are signified by the red points on the Figure. Figures 3-6 demonstrate the evolution of the hybrid algorithm for the four sub-populations. Each Figure presents the evolution of a subpopulation.

From the Figures for the evolution of the subpopulations when the algorithm was deployed, it can be observed that both mean fitness and best fitness indices are relatively high at initialization, but decreased steadily as the number of generations increases. The fall in fitness indices with an increase in generations implies an emergency of better solutions since the required optimization is a minimization problem. In fact, on deployment of NSGA-II alone, the fitness indices started at around $4 X 10^{5} \mathrm{dBm}$ on initialization for the interference cost function and declined to $1.5 \times 10^{5} \mathrm{dBm}$ at the end of $100^{\text {th }}$ generation (Figures 1a). However, the fitness indices started between $6 \times 10^{3} \mathrm{dBm}$ and $1 X 10^{3} \mathrm{dBm}$ at initialization and decreased to a value below 200 at the end of the $100^{\text {th }}$ generation for the demand infringement objective function (Figure 1b). The trend is similar throughout but greatly improved at hybridization.

The Performance of the hybrid evolutionary algorithm-artificial neural network for optimum frequency allocation in a heterogeneous frequency requisition network is as presented in Table 2. As indicated in the table; the minimum interference objective function has mean fitness and best fitness of 142530 and 144170 respectively at the deployment of NSGA-II alone. At the same instance, mean fitness and best fitness of 196 and 162 were obtained for the demand infringement objective function. However, the application of the hybrid produced better results. Mean fitness and best fitness of 134100 and 122920 respectively were achieved for the minimum interference objective function. The demand infringement objective function has 162 and 158 for mean and best fitness respectively 

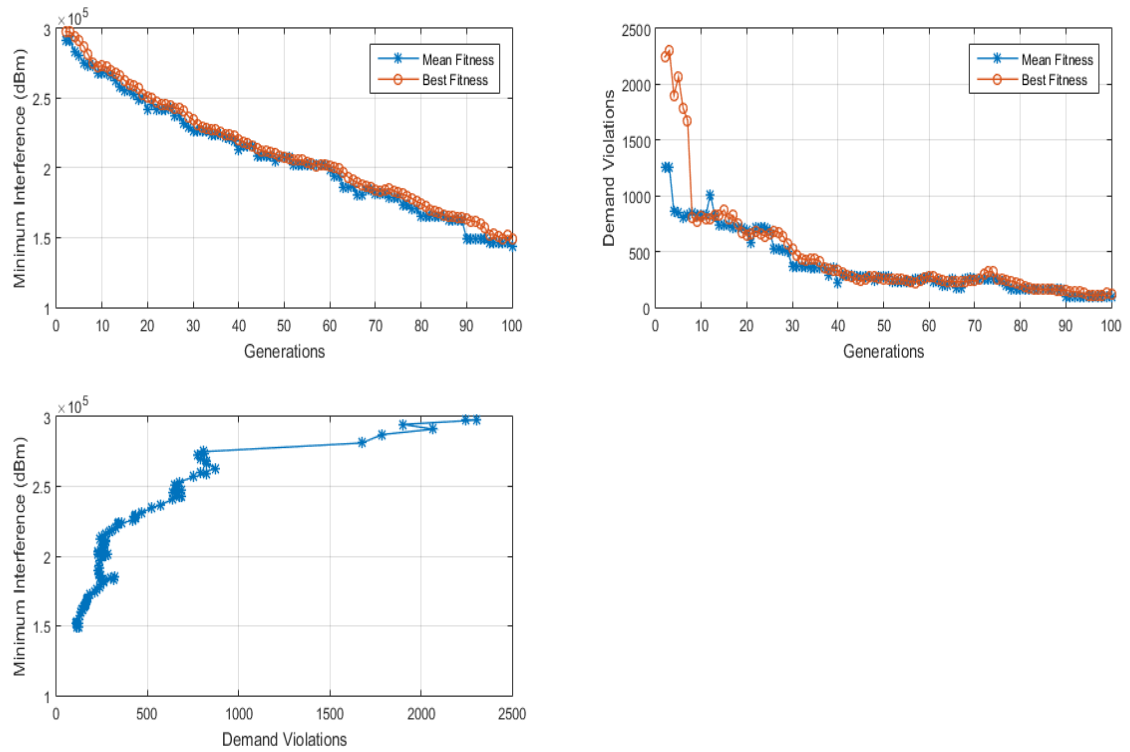

Figure 3: Evolution of the sub-population 1 when the hybrid algorithm was deployed; (a) Evolution of the minimum interference; (b) Evolution of the demand infringement; (c) Combination of the minimum interference $\&$ the demand infringement.
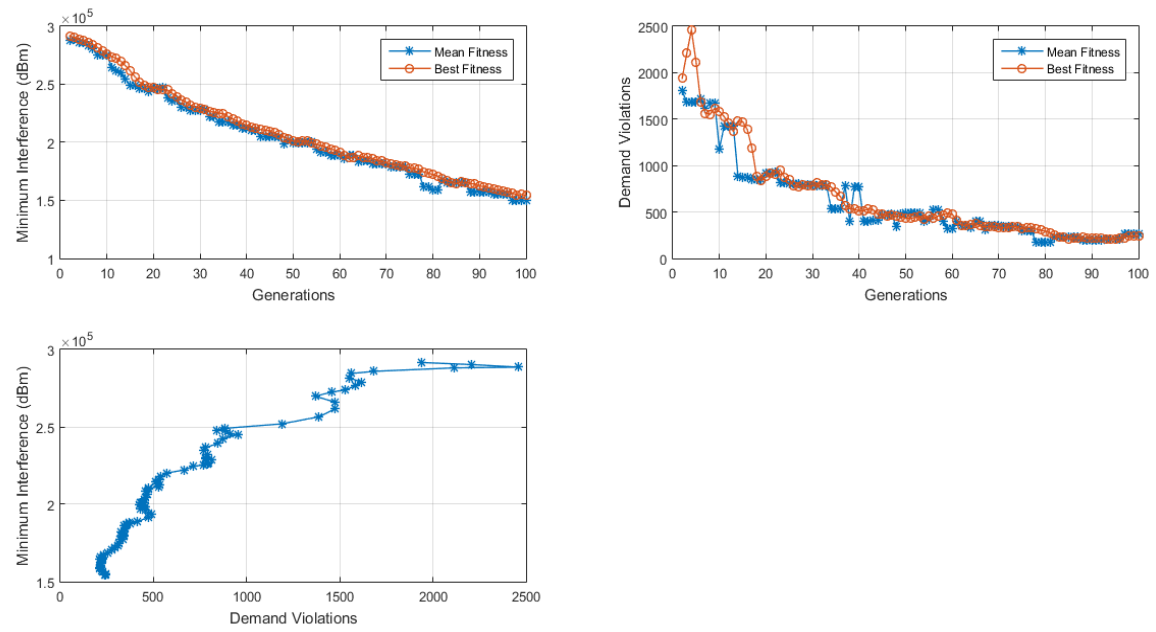

Figure 4: Evolution of the sub-population 2 when the hybrid algorithm was deployed; (a) Evolution of the minimum interference; (b) Evolution of the demand infringement; (c) Combination of the minimum interference $\&$ the demand infringement. 

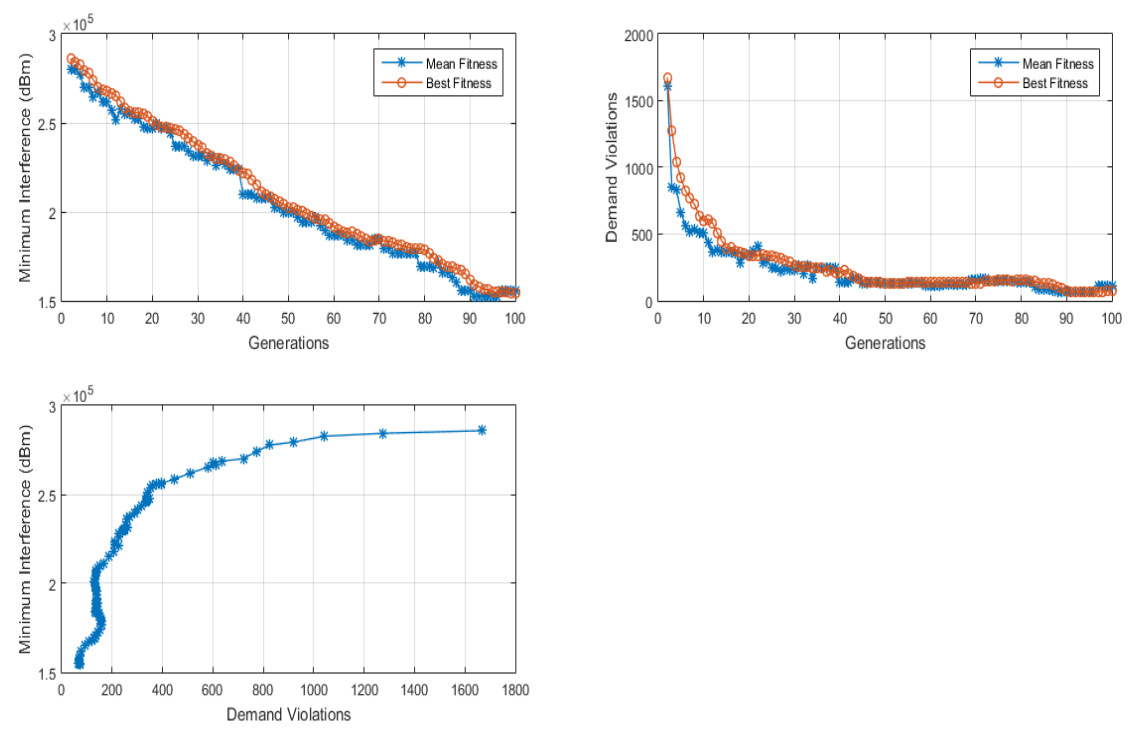

Figure 5: Evolution of the sub-population 3 when the hybrid algorithm was deployed; (a) Evolution of the minimum interference; (b) Evolution of the demand infringement; (c) Combination of the minimum interference \& the demand infringement.
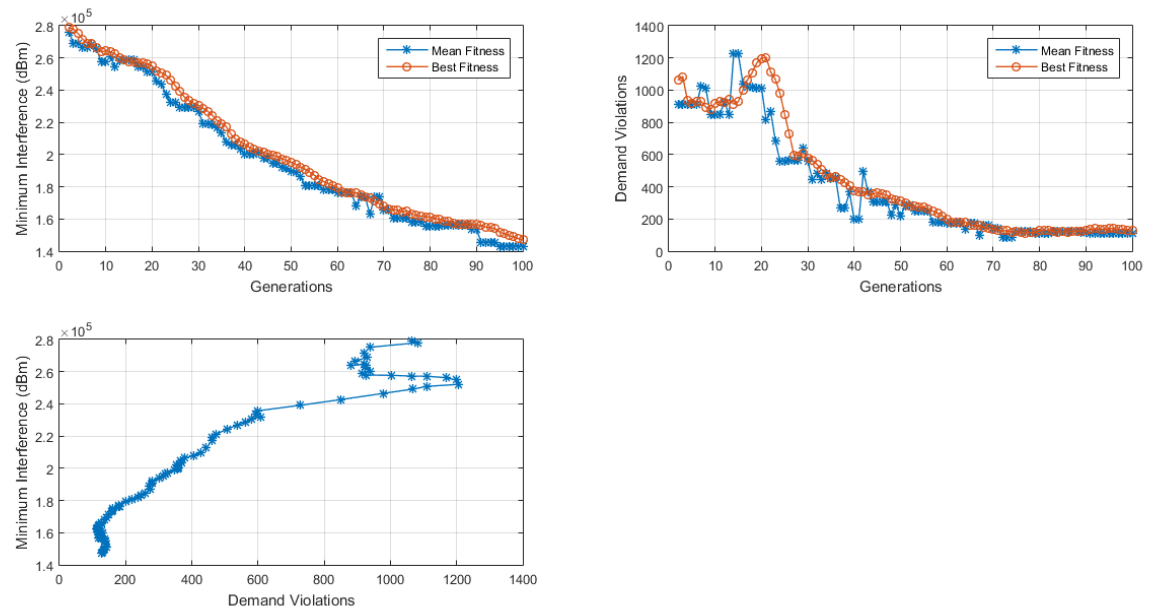

Figure 6: Evolution of the sub-population 4 when the hybrid algorithm was deployed; (a) Evolution of the minimum interference; (b) Evolution of the demand infringement; (c) Combination of the minimum interference $\&$ the demand infringement. 
Table 2: Performance of the hybrid Algorithm in a Heterogeneous Frequency Requisition Network

\begin{tabular}{|c|c|c|c|c|c|c|}
\hline \multirow{2}{*}{$\begin{array}{l}\text { The Performance } \\
\text { Evaluation Metrics }\end{array}$} & \multicolumn{2}{|l|}{ NSGA-II } & \multicolumn{2}{|c|}{$\begin{array}{l}\text { Hybrid Algorithm } \\
\text { (NSGA-II \& SOM) }\end{array}$} & \multicolumn{2}{|c|}{ Percentage Improvement } \\
\hline & $\begin{array}{l}\text { Interference } \\
{[D(A)]}\end{array}$ & Demand $[d(A)]$ & $\begin{array}{l}\text { Interference } \\
{[\rho(A)]}\end{array}$ & $\begin{array}{l}\text { Demand } \\
{[d(A)]}\end{array}$ & $\begin{array}{l}\text { Interference } \\
{[\rho(A)]}\end{array}$ & Demand $[d(A)]$ \\
\hline Mean Fitness & 142530 & 196 & 134100 & 162 & $6 \%$ & $17 \%$ \\
\hline Best Fitness & 144170 & 162 & 122920 & 158 & $15 \%$ & $3 \%$ \\
\hline
\end{tabular}

The percentage enhancement in the performance of the hybrid algorithm to effectively allot in terms of fitness indices is as presented in Table 2. For the minimum interference objective function, the performance improvements were $6 \%$ and $15 \%$ respectively for the mean score fitness and best score fitness indices. In the case of the demand infringement objective function, the performance improvements were $17 \%$ and $3 \%$ respectively for the mean score and best score fitness indices when the hybrid algorithm was deployed

\section{Conclusion}

In this study, hybrid "evolutionary algorithmartificial neural network" was deployed for optimum real time frequency allocation in a heterogeneous frequency requisition network to ensuring minimized inter-cell interference. From the results obtained, based on fitness values, the hybrid algorithm gave an improved performance, thereby providing an optimal frequency allocation that minimizes interference. The algorithm can be embedded in software applications for real-time optimal resources allocation in cellular networks.

\section{References}

[1] Lasisi, H., Oyedeji, M.O. and Adediran, Y.A. "Application of Hybrid Non-Dominated Sorting Genetic Algorithm-II and SelfOrganizing Map Technique to Interference Management in Homogeneous Frequency Demand Cellular Network", IEEE $3^{\text {rd }}$ International Conference On Electro-Technology For
[2] Yang Shengxiang, C.H. and F.W. "Dynamic Genetic Algorithms with HyperMutation Schemes for Dynamic Shortest Path Routing Problem in Mobile Ad Hoc Networks", IEEE Transactions on Systems, man and cybernetics, vol. 40, no. 1, 2010, pp. 52-63. doi:10.4018/jaras.2012010105

[3] Cheng, H., Yang, S. and Cao, J. "Dynamic Genetic Algorithms for the Dynamic Load Balanced Clustering Problem in Mobile ad hoc Networks", Expert Systems with Applications, vol. 40, no. 4, 2013, pp. 1381-1392. doi:10.1016/j.eswa.2012.08.050

[4] Pinagapany, S. and Kulkarni, A.V. "Solving Channel Allocation Problem in Cellular Radio Networks using Genetic Algorithm", 3rd IEEE/Create-Net International Conference on Communication System Software and Middleware, COMSW ARE, 2008, pp. 239-244. doi:10.1109/COMSWA.2008.4554416

[5] Ekbatanifard, G.H., Monsefi, R., Akbarzadeh-T., M.R. and Yaghmaee, M.H. "A Multi-objective Genetic Algorithm-based Approach for Energy-efficient QoS-routing in Two-tiered Wireless Sensor Networks", ISWPC 2010 - IEEE 5th International Symposium on Wireless Pervasive Computing, 2010, pp. 80-85. doi:10.1109/ISWPC.2010.5483775

[6] Zhang, Y.D. and Wu, L.N. "A Novel Genetic Ant Colony Algorithm", Journal of convergence information technology, vol. 7, no. 1, 2012, pp.268-274. doi:10.1155/2016/2167413

[7] Lorenzo, B. and Glisic, S. "Optimal Routing and Traffic Scheduling for Multihop 
Cellular Networks using a Genetic Algorithm", IEEE Transactions on Mobile Computing, vol. 12, no. 11, 2013, pp. 22742288. doi:10.1109/TMC.2012.204

[8] Niu, S.H., Ong, S.K., and Nee, A.Y.C. "An Enhanced Ant Colony Optimizer for Multi-attribute Partner Selection in Virtual Enterprises", International Journal of Production Research, vol. 50, no. 8, 2012 pp. 2286-2303. doi:10.1080/00207543.2011.578158

[9] Srininvas N. and Deb. K. "Multiobjective Optimization using Nondominated Sorting in Genetic Algorithms. Evolution and computation, vol.2, no. 3, 1995, pp. 221-248.

[10] Deb. K., Agrawal, S., Pratap, A. and Meyarivan, M.T. "A Fast and Elitist Multiobjective Genetic Algorithm": NSGA-II, vol. 6, no. 2, 2002, pp. 182-197. doi:10.1109/4235.996017

[11] Hans, A.E. "Multicriteria Optimization for Highly Accurate Systems. Multicriteria optimization in Engineering and Sciences". New York: Mathematical Concepts and Methods in Science and Engineering,19, 1988 pp. 309-352. New York: Plenum Press.

[12] Dorne, R. and Jin-Kao Hao, J.K. "An Evolutionary Approach for Frequency Assignment in Cellular Radio Networks", IEEE International Conference on Evolutionary Computation (ICEC'95), vol. 2, 1995, pp. 539544.

[13] Kotrotsos, S., Kotsakis, G., Demestichas, P., Tzifa, E. and Demesticha, V. "The self-organizing map", Proceedings of the IEEE, vol. 78, no. 9, 1990, pp. 1464-1480. doi: $10.1109 / 5.58325$

[14] Lasisi, H., Adediran, Y.A. and Ayodele, A.A. "Development of Propagation Path Loss Prediction Model for Mobile
Communications Network Deployment in Osogbo, Nigeria", EJERS, European Journal of Engineering Research and Science, vol. 2, no. 11, 2017, pp. 13-17. http://www.ejers.org 